\title{
Keeping up with the times: how national public health and governmental organizations communicate about cannabis on Twitter
}

\author{
Jenna van Draanen ${ }^{1 *}$ (D) Tanvi Krishna ${ }^{2}$, Christie Tsang ${ }^{3}$ and Sam Liu ${ }^{4}$
}

\begin{abstract}
Background: Public health and governmental organizations are expected to provide guidance to the public on emerging health issues in accessible formats. It is, therefore, important to examine how such organizations are discussing cannabis online and the information that is being provided to the public about this increasingly legal and available substance.

Methods: This paper presents a concise thematic analysis of both the volume and content of cannabis-related health information from selected $(n=13)$ national-level public health and governmental organizations in Canada and the U.S. on Twitter.

Results: There were eight themes identified in Tweets including 1) health-related topics; 2) legalization and legislation; 3) research on cannabis; 4) special populations; 5) driving and cannabis; 6) population issues; 7) medical cannabis, and 8) public health issues. The majority of cannabis-related Tweets from the organizations studied came from relatively few organizations and there were substantial differences between the topics covered by U.S. and Canadian organizations. The organizations studied provided limited information regarding how to use cannabis in ways that will minimize health-related harms.

Conclusions: Authoritative organizations that deal with public health may consider designing timely social media communications with emerging cannabis-related information, to benefit a general public otherwise exposed to primarily pro-cannabis content on Twitter.
\end{abstract}

Keywords: Cannabis, Marijuana, Messaging, Education, Public health, Social media

\section{Background}

Although the platform is just over 10 years old, Twitter has significantly changed the way we interact with media. Twitter allows users to produce and consume information in 280-character segments (revised from 140 characters in 2017); with posts ranging from updates on important current events to mundane personal musings, it has changed the way in which we intake all types of information and given us the ability to connect with others instantaneously.

\footnotetext{
* Correspondence: jennavandraanen@gmail.com

${ }^{1}$ Faculty of Arts, Department of Sociology, University of British Columbia, Vancouver, BC, Canada

Full list of author information is available at the end of the article
}

These messages, or Tweets, have been used to analyze public opinion on a range of health topics. [1-4]

The public health community uses Twitter occasionally for the spread of health information. [5, 6] Advantages of spreading public health information this way include limited costs, wide audiences, and timely information exchange, but these come at the cost of perceived lack of credibility and difficulty for the audience in distinguishing opinion from evidence. [6] Paul and Dredze studied state public health department adoption of social media and found that $60 \%$ of public health departments in the United States (U.S.) were using at least one type of social media; among these, $86.7 \%$ had a Twitter account [5]. Public health departments had an average of 983 Twitter

(c) The Author(s). 2019 Open Access This article is distributed under the terms of the Creative Commons Attribution 4.0 International License (http://creativecommons.org/licenses/by/4.0/), which permits unrestricted use, distribution, and 
followers, although there was considerable variety in the reach of messages, depending on the organization [5]. On average, state public health departments tended to post on social media only once per day, and substance use was not among the topics commonly posted [5].

In both Canada and the U.S., there has been considerable policy change recently, regarding the decriminalization and even legalization of cannabis. Several U.S. states have passed legislation to legalize recreational use of cannabis (e.g., Colorado, Washington, California) and the federal government in Canada has done the same. The continental landscape of cannabis policy is shifting, as is cannabis discussion online. Thompson and colleagues assessed cannabis-related Tweets posted by adolescents in 2012 and found that a majority (65.6\%) of Tweets by adolescents reflected a positive attitude toward cannabis, and $42.9 \%$ indicated personal use. [7] Both Tweets about personal cannabis use and positive perceptions about cannabis increased from 2012 to 2013. [7] Cavazos-Rehg and colleagues analyzed the sentiment of a random sample of Tweets related to cannabis in 2014 and, again, found that most had a positive sentiment towards use, with pro-cannabis Tweets outnumbering anticannabis Tweets by a factor of 15. [8]

Public health and governmental organizations are expected to provide guidance to the public on emerging health issues, even in the absence of perfect information about risks and benefits. It is, therefore, important to examine how such organizations are discussing cannabis online and the information that is being provided to individuals who need to navigate safe use or decisions related to non-use of this increasingly legal and available substance. This paper provides a concise analysis of both the volume and content of cannabis-related information from selected public health organizations in Canada and the U.S. on Twitter.

\section{Methods}

\section{Sampling and data collection}

A total of $n=41,600$ Tweets were collected in September 2017 using the Twitter application programming interface (API), were stored in CSV files, and were analyzed using Dedoose Software. [9] Public health and mental health/substance use organizations in Canada and the U.S. were eligible for inclusion in the analysis if they met the following criteria: had an active Twitter account (posted at least once per month in the previous 12-month period), provided national services or had a national scope/mandate of their work, were either a governmental organization or a notfor-profit organization, and posted a Tweet at least once in the last 12 month period about mental health or substance use issues.

The screening criteria yielded the following organizations in Canada for inclusion in our dataset: The Public Health Agency of Canada (PHAC) @PHAC_GC, Health
Canada (HC) @GovCanHealth, the Canadian Public Health Association (CPHA) @CPHA_ACSP , The Canadian Mental Health Association (CMHA) @CMHA NTL, the Mental Health Commission of Canada (MHCC) @MHCC_, the Canadian Institutes of Health Research (CIHR) @CIHR_IRSC, and the Centre for Addiction and Mental Health (CAMH) @CAMHNews; and the following organizations in the U.S.: The Department of Health and Human Services (DHHS) @HHSGov, The National Institute on Drug Abuse (NIDA) @NIDAnews, The Centres for Disease Control (CDC) @CDCgov, the Substance Abuse and Mental Health Services Administration (SAMHSA) @samhsagov, the American Public Health Association (APHA) @PublicHealth, the National Alliance on Mental Illness (NAMI) @NAMICommunicate, and the National Institutes of Health (NIH) @NIH.

This study is not intended to provide a comprehensive inventory of all public health organization activity on Twitter. Instead, key national organizations $(n=13)$ were selected with the intent of offering descriptive information about the volume and content of their public health messaging related to cannabis consumption. A total of 3200 of the most recent Tweets per organization (resulting in a total sample of 41,600 Tweets) were selected to be included in the initial dataset. For some organizations, based on the frequency of their Tweets, this meant that the period of analysis went back as far as 2011, for others it only went back to 2015. Keyword searches were conducted on this dataset to retain only the Tweets relevant to cannabis that contained any of the terms: 'weed,' 'THC,' 'cannabis,' 'marijuana,' 'medical marijuana,' or 'pot,' yielding the intermediate datasets depicted in Table 1 . From this pool, reTweets were excluded and researchers hand-sorted Tweets to identify the ones relevant for analysis, producing the final datasets depicted in Table 1 . The data used in this study are public and as such this study is exempt from human subject's review.

\section{Data analysis}

Data were analyzed using thematic content analysis. First, two researchers open-coded a selection of 50 Tweets and generated a codebook to be used in the first round of focused coding. Next, during focused coding, each researcher coded 100 Tweets according to these initial codes. After this initial focused coding, the researchers met to discuss and refine the themes according to emerging sub-themes. This revised codebook was then used to classify all Tweets, with each Tweet categorized by two researchers, after which the researchers again met to resolve disagreements in coding. Tweets were allowed to be classified in more than one thematic area where appropriate. Inter-rater reliability was calculated using a random sample of 100 Tweets, using Dedoose software [9] and the pooled Kappa 
Table 1 Screening and Resulting Sample Size of Cannabis-related Datasert for Included Organizations

\begin{tabular}{|c|c|c|c|c|c|c|c|c|c|c|c|c|c|c|}
\hline \multirow[b]{2}{*}{ Organization Name } & \multicolumn{7}{|c|}{ Canadian Organizations } & \multicolumn{7}{|c|}{ U.S. Organizations } \\
\hline & $\mathrm{CPHA}$ & $\mathrm{CAMH}$ & $\mathrm{HC}$ & $\mathrm{CMHA}$ & PHAC & $\mathrm{MHCC}$ & $\mathrm{ClHR}$ & NIDA & SAMHSA & $\mathrm{NIH}$ & APHA & NAMI & $\mathrm{CDC}$ & $\mathrm{DHHS}$ \\
\hline \multirow[t]{2}{*}{ Starting dataset (n) } & 3200 & 3200 & 3200 & 3200 & 3200 & 3200 & 3200 & 3200 & 3200 & 3200 & 3200 & 3200 & 3200 & 3200 \\
\hline & $\downarrow$ & $\downarrow$ & $\downarrow$ & $\downarrow$ & $\downarrow$ & $\downarrow$ & $\downarrow$ & $\downarrow$ & $\downarrow$ & $\downarrow$ & $\downarrow$ & $\downarrow$ & $\downarrow$ & $\downarrow$ \\
\hline \multirow[t]{2}{*}{ Dataset after keyword filter (n) } & 200 & 158 & 81 & 50 & 117 & 143 & 87 & 345 & 114 & 120 & 17 & 55 & 134 & 276 \\
\hline & $\downarrow$ & $\downarrow$ & $\downarrow$ & $\downarrow$ & $\downarrow$ & $\downarrow$ & $\downarrow$ & $\downarrow$ & $\downarrow$ & $\downarrow$ & $\downarrow$ & $\downarrow$ & $\downarrow$ & $\downarrow$ \\
\hline $\begin{array}{l}\text { Dataset after RT removal and irrelevant } \\
\text { Tweets removed (n) }\end{array}$ & 108 & 84 & 47 & 5 & 4 & 1 & 5 & 291 & 14 & 3 & 17 & 0 & 0 & 1 \\
\hline
\end{tabular}

statistic was found to be 0.94 [10] indicating excellent reliability. [11]

\section{Results}

The majority of cannabis-related Tweets from the organizations studied come from relatively few organizations: CAMH (84/3200), CPHA (108/3200), and NIDA (291/ $3200)$. See Table 2 for a summary of the volume of Tweets, date range of included data, number of followers for each included organization, and the thematic content of their cannabis-related Tweets. The organizations with the most followers were the CDC $(863,534)$, the NIH (811, 577), and the DHHS (711,590); notably, they had few (4) cannabis-related Tweets combined. Tweets from the included health organizations were primarily informationsharing Tweets with links to research results, guidelines, policies, or fact sheets.

There were a total of eight themes found in the coded dataset, listed and described in order of frequency below. Twenty-three subthemes were present within the main themes and have been identified with bold font in the theme descriptions below.

\section{T1: health-related topics}

One hundred three Tweets (or close to $18 \%$ of the 580 Tweets in the dataset) contained themes related to human health, most commonly about the neurological issues or brain changes that can arise when using cannabis (49 Tweets). However, within this theme, subthemes of beneficial or therapeutic effects of cannabis use, the impact of cannabis on the development of mental illness or effects of using cannabis for people with psychiatric disorders, the relationship between cannabis use and substance use disorders, and lastly smoking (tobacco and cannabis) were also found. Examples of health-related Tweets about neurological issues include the National Institutes of Health Tweet, "Cannabis can be addictive $\mathcal{E}$ has negative effects on attention, memory, \& learning: https://t.c \#MTF2015"and the Centre for Addiction and Mental Health Tweet, "Quitting cannabis use improves \#cognition in people with schizophrenia." Notably, as Tweets were allowed to be coded into more than one category, the CAMH Tweet example above was also coded in the cannabis and mental illness subtheme. Health-related topics were Tweeted about relatively more frequently by U.S. organizations than Canadian organizations.

\section{T2: legalization and legislation}

Nearly one in five Tweets analyzed (103 Tweets, or 18\%) were related to legalization of cannabis and/or cannabis-related legislation. Most frequently (23 Tweets), the social media activity was promoting legalization along with careful regulation of the substance; or (22 Tweets) involved experts such as substance use researchers or policy makers offering expert opinions on legalization. Compared to U.S.based organizations, Canadian health organizations Tweeted more frequently about legalization, regulation, and legislation, driving most of the content within this theme (as might be expected given recent federal policy changes in Canada). An example of a representative Tweet from this theme, from Health Canada is, "Have your say on legalization $\mathcal{E}$ regulation of \#cannabis https://t.c..." Other subthemes in this category included: discussions about the issues with legalization (for example, how to restrict access for minors); promoting proposed legislation, which usually involved discussion about a particular bill or law; governmental announcements about policy change; and advertising upcoming public consultations about cannabis regulation.

\section{T3: research on cannabis}

Research on cannabis was highlighted and referenced in many posts (101 Tweets or 17\%), for example, the National Institute on Drug Abuse shared a Tweet, "What are cannabis's long-term effects on the brain? To find out, see our updated Cannabis Research Report. https://t.c." Most commonly, the post was generally designed to publicize research results (67 Tweets) and referenced a new paper, study, or report, without describing any direct application to consumers. Research also commonly highlighted interactions between cannabis and alcohol (24 Tweets) or cannabis and opioids (9 Tweets). A small number of Tweets described financial and legislative barriers to research and the way that legalization would make research more feasible. 
Table 2 Volume of Cannabis-related Tweets, Date Range Studied, Number of Followers, and Frequency of Themes in Tweets from Included Public Health and Governmental Organizations

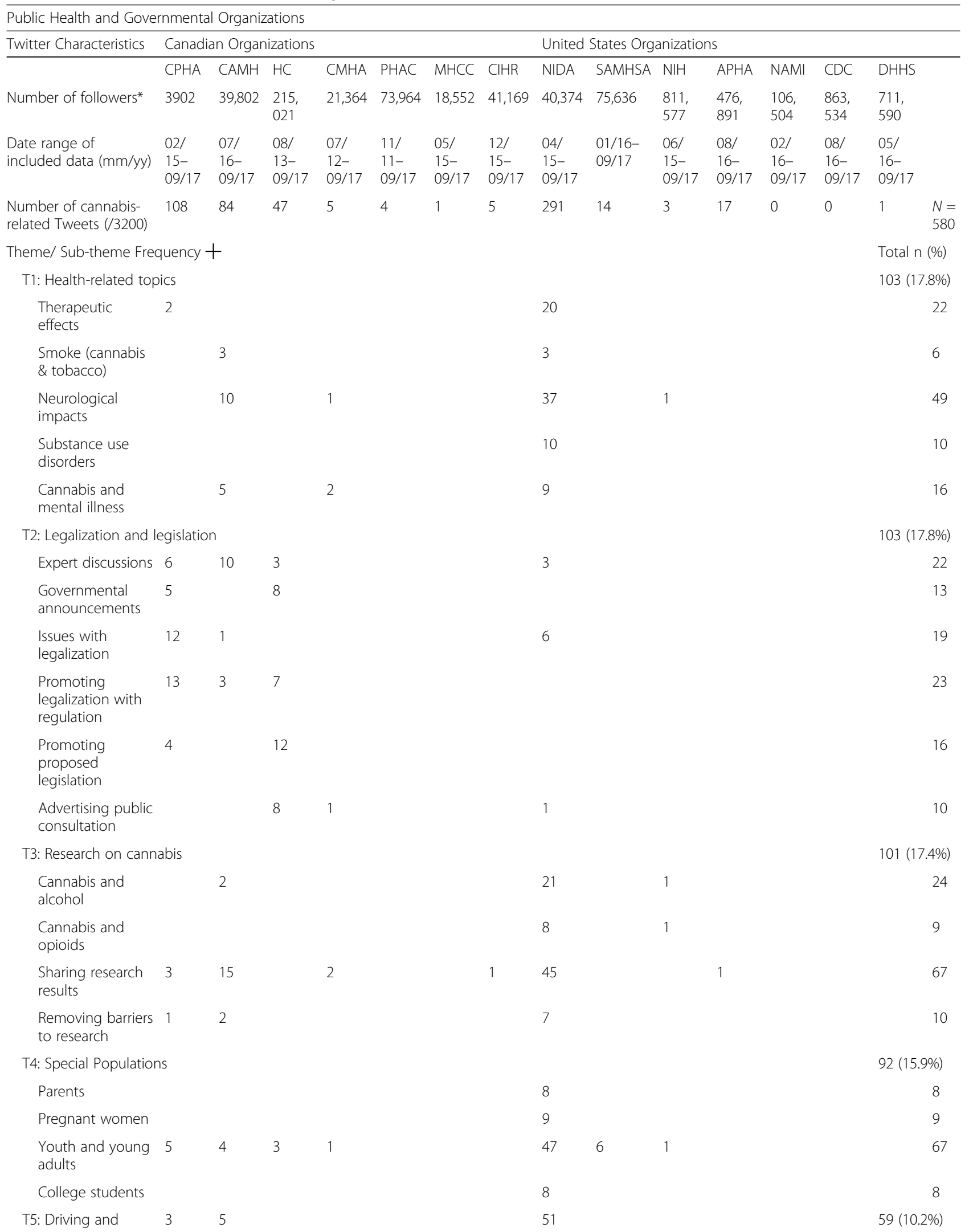


Table 2 Volume of Cannabis-related Tweets, Date Range Studied, Number of Followers, and Frequency of Themes in Tweets from Included Public Health and Governmental Organizations (Continued)

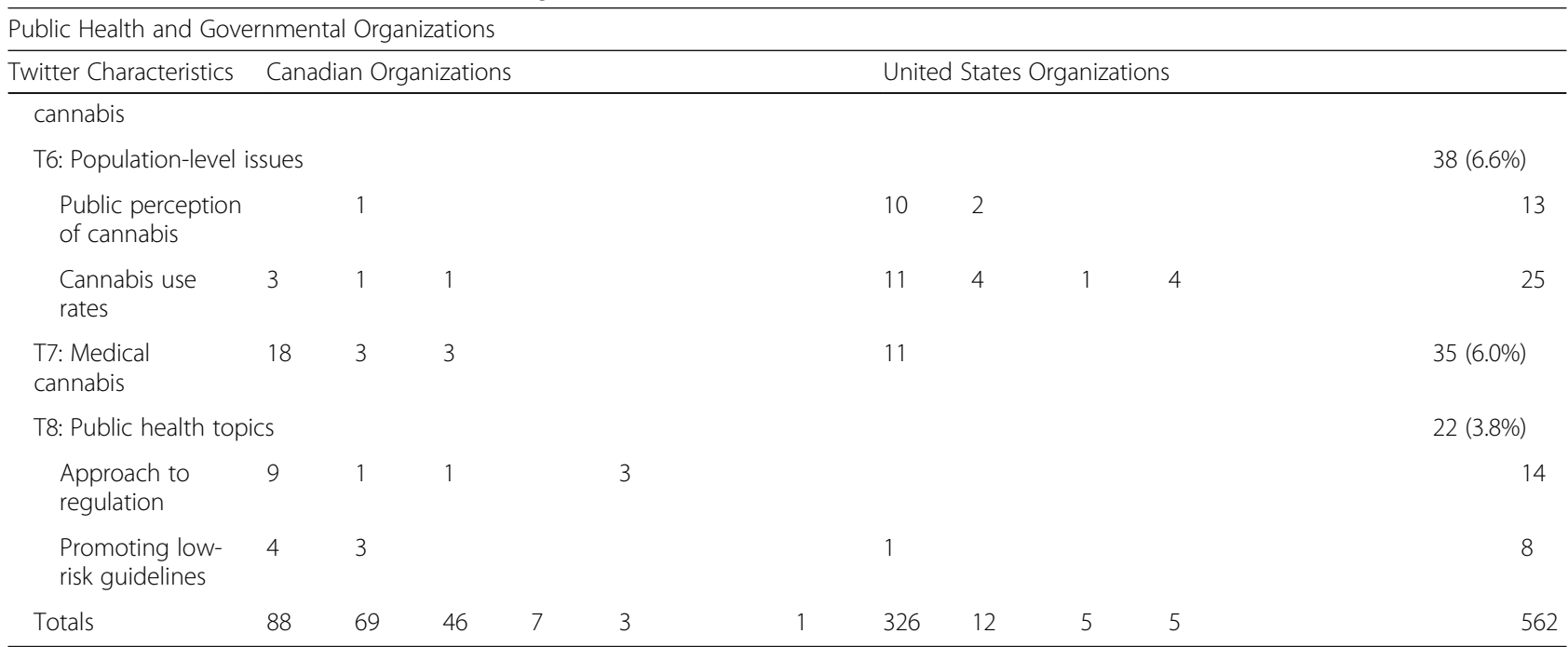

† Tweets that did not fall in any existing themes or subthemes were included in an "other" category not depicted here, coding more than one category per Tweet was allowed

*Number of followers as of Oct 19, 2017

Highlighting research results via Twitter was done more commonly by U.S. than Canadian organizations.

\section{T4: special populations}

Special populations were also mentioned frequently in Tweets (92 Tweets or 16\%) usually with the Tweets discussing prevalence rates or unique considerations for cannabis use within particular populations, including youth and young adults, pregnant women, parents, and college students. Youth and young adults were by far the most commonly referenced population group, for example, SAMHSA Tweeted "Acting Dir Baum: 20.8\% of 18-25 year olds reported past month \#cannabis use. Highest rate ever over past 15 years. \#recoverymonth."

\section{T5: driving and cannabis}

Just over $10 \%$ of the Tweets analyzed contained information about the effect that cannabis has on driving and warnings about driving while or after using cannabis. There was limited variation in the nearly 60 Tweets in this category, and as such there were no sub-themes. NIDA, a U.S. Organization, was responsible for the majority of Tweets in this category, Tweeting, for example, "Dr. Marilyn Huestis discusses how \#marijuana impairs a measure of driving. https://t.co/FC4mgq6j3w"

\section{T6: population-level issues}

Nearly 7\% of the Tweets about cannabis by public health and governmental organizations were concerning issues at the population level, like cannabis use rates and public opinion or perception of cannabis. These population- wide topics came up more frequently in U.S. than Canadian organizations.

\section{T7: medical cannabis}

Using cannabis for medicinal purposes, and Tweets that were about therapeutic use (vs. recreational use) or medical marijuana licensure or prescription were contained in a medical cannabis theme that included $6 \%$ of the total Tweets. This theme did not have sufficient variety to warrant subthemes. An example of a Tweet in this theme comes from CAMH, "New research from $\mathrm{CAMH}$ : Medical marijuana programs and implications for cannabis control policy http://t.co/SOgyzvOxI3".

\section{T8: public health topics}

The least common theme, containing 22 Tweets, was public health topics. Content related to health outcomes and medical issues were captured in T1, and as such, most of the explicit public health content from the organizations concerned public health approaches to regulation and sharing low-risk guidelines for cannabis use to minimize harm. For example, the Public Health Agency of Canada Tweeted, "\#GoC committed to comprehensive \#publichealth education on impacts of \#cannabis. https://t.co/WdxDFyqH4P".

\section{Discussion}

The organizations studied provided limited consumer-focused information regarding how to use cannabis in ways that will minimize health-related harms. Although the content of the Tweets did often relate to health issues (18\% of 
Tweets), the nature of the Tweets in the dataset was primarily of a promotional or information-sharing style and rarely included specific advice, education, or instruction for followers. Given the pro-cannabis sentiment of much of the cannabis-related Tweets from the general public [12], Twitter users would benefit from more public-health driven content containing educational information related to health impacts of cannabis use. Some examples of educational messages designed to target the general public and to help maximize safety and health when using cannabis include, "start low and go slow" as well as, "use cannabis in a safe and familiar environment with people you trust" and, "if you are a new consumer, look for a product with less than $100 \mathrm{mg} / \mathrm{g}(10 \%) \mathrm{THC}$, with equal or higher levels of CBD," etc. [13].

The use of cannabis for recreational purposes remains illegal federally in the United States, with some states adopting laws that permit legal recreational and/or medical use. As of July 2019, 11 states in the U.S. had passed laws permitting the use of cannabis for adults, whereas at the end of the period of data collection for this study there were 8 states where use was legal [14]. In Canada, a national approach to legalization was taken, with the use of cannabis for recreational purposes becoming legal on October 17, 2018 [15]. These different approaches to legalization undoubtedly affect how the organizations in our sample communicate with the public about cannabis use.

While health-related topics, population-level issues, and Tweets about research on cannabis were more common in the U.S. organizations studied, Tweeting about legislation, legalization, and regulation were more common in the Canadian organizations. It is possible that the impending policy change to legalize cannabis in Canada (at the time period studied) offered a unique policy window where non-governmental organizations could propose benefits of legalization and approaches to regulation that would be considered by policy makers, and where governmental organizations could advertise consultations and public dialogue about cannabis without fear of promoting criminalized activity, making discussion about legislative changes both more timely and relevant. In the absence of legislative change at the national level or indications of consideration of a national policy change, the U.S. organizations studied may have opted to Tweet information about other topics more salient to their followers and less politically objectionable. When approaching legalization, though, health-related topics become increasingly relevant to the general public and the relatively lower frequency of posting about health outcomes in Canadian organizations may represent a critical absence of useful information for Canadian Twitter users. Further, legalization is in itself a public health issue, and one that non-governmental organizations could be advancing discussion of, even without any actual or impending policy decisions from government.
Based on our findings, it appears that in both countries public health and governmental organizations are not capitalizing on social media's interactive potential and could be providing more direct information that is related to what Twitter users are discussing. [7] The organizations included in our study rarely posted about cannabis on Twitter, with the exception of NIDA, CAMH, CPHA, and HC and when they did post cannabis-related content it was primarily sharing research findings, reports, or links that contain information such as issues for youth and young adults, driving and cannabis use, neurological impacts of cannabis use, and medical cannabis.

There are other substances that have a heavily imbalanced "pro use" presence on social media, such as alcohol $[16,17]$, and this warrants further discussion as the public may benefit from public health and mental health/substance use organizations offering more information about how to use a variety of substances in ways that maximize health and safety, not just cannabis. Future research investigating other substance use-related content from health organizations may be needed. In addition, investigating how social media strategy is crafted and to what aim within such organizations is warranted.

Paul and Dredze report findings about the interactions between public health departments and Twitter users. [5] The departments in their study posted on average once per day and re-Tweets constituted $22.5 \%$ of all their Tweets; only $1.5 \%$ of Tweets were in response to a Tweet made by a follower. [5] This one-way social media communication pattern represents a missed opportunity to engage with Twitter users about information they may be seeking. Public health and governmental organizations would be well advised to incorporate social media into their substance use communication strategy, and given the recent switch to allow up to 280 characters on Twitter, this may be more feasible than it was previously. $[5,18]$

\section{Limitations}

Health organizations were selected by searching the internet for representative organizations that met search criteria and by identifying an institutional account from each organization's web page. It is possible that individual programs or units within an organization are also using Twitter and were not identified. It is also possible that other public health agencies and non-governmental organizations not included in the sample were Tweeting relevant content that was not captured. Thus, the results of this analysis should not be extrapolated to institutions beyond the scope of this study. Future research should be designed to examine state or provincial Twitter activity to better understand regional differences in public health education messages about cannabis on Twitter. Moreover, this analysis does not allow us to comment on cannabis conversation on other social media platforms or public health education campaigns that may 
occur outside of Twitter: another avenue worth exploring in future research.

\section{Conclusions}

Public health and governmental organization are tentative or occasional Twitter users with respect to using Twitter as a venue for communicating cannabis-related health information. As social media is becoming ever more pervasive, these organizations should consider ways to use Twitter to engage their audiences and create relationships which are usually hindered by budget and geographic restrictions. Given the pro-cannabis sentiment of the cannabis-related Tweets from the general public, Twitter users would benefit from more targeted public health-driven content containing educational information related to health impacts of cannabis use.

\section{Abbreviations}

APHA: American Public Health Association; $\mathrm{CAMH}$ : Centre for Addiction and Mental Health; CBD: Cannabidiol; CDC: Centres for Disease Control; CMHA: Canadian Mental Health Association; CPHA: Canadian Public Health Association; HC: Health Canada; DHHS: Department of Health and Human Services; MHCC: Mental Health Commission of Canada; NAMI : National Alliance on Mental IIIness; NIDA: National Institute on Drug Abuse; $\mathrm{NIH}$ : National Institutes of Health; PHAC: Public Health Agency of Canada; SAMHSA: Substance Abuse and Mental Health Services Administration; THC: Tetrahydrocannabinol; U.S.: United States

\section{Acknowledgements}

Not applicable.

\section{Authors' contributions}

JVD conceptualized the study, analyzed data, and was the main contributor in writing the manuscript. CT and TK analyzed data and assisted with manuscript preparation. SL collected data, provided methodological guidance, and was a major contributor in writing the manuscript. All authors read and approved the final manuscript.

\section{Funding}

Dr. van Draanen was supported by a CIHR Postdoctoral Fellowship and a Michael Smith Foundation for Health Research Trainee Award.

\section{Availability of data and materials}

The datasets generated and/or analysed during the current study are available in the Twitter API repository, https://developer.twitter.com/

\section{Ethics approval and consent to participate}

The data used in this study are public and as such this study is exempt from human subject's review.

\section{Consent for publication}

Not applicable.

\section{Competing interests}

The authors declare that they have no competing interests.

\section{Author details}

${ }^{1}$ Faculty of Arts, Department of Sociology, University of British Columbia, Vancouver, BC, Canada. ${ }^{2}$ Faculty of Arts, Department of Psychology, University of British Columbia, Vancouver, BC, Canada. ${ }^{3}$ Faculty of Arts, School of Social Work, University of British Columbia, Vancouver, BC, Canada. ${ }^{4}$ Faculty of Education, School of Exercise Science, Physical \& Health Education, University of Victoria, Victoria, BC, Canada.
Received: 25 March 2019 Accepted: 21 August 2019

Published online: 12 September 2019

References

1. Chew C, Eysenbach G. Pandemics in the age of twitter: content analysis of tweets during the 2009 H1N1 outbreak. PLoS One. 2010;5(11):e14118.

2. Scanfeld D, Scanfeld V, Larson EL. Dissemination of health information through social networks: twitter and antibiotics. Am J Infect Control. 2010; 38(3):182-8.

3. Liu S, Miaoqi Z, Young S. Monitoring freshman college experience through content analysis of tweets: observational study. JMIR Public Health Surveill. 2018;4(1):e5. https://doi.org/10.2196/publichealth.7444.

4. Liu S, Young S. A survey of social media data analysis for physical activity surveillance. J Forensic and Leg Med. 2018;57:33-6.

5. Paul MJ, Dredze M. You are what you Tweet: Analyzing Twitter for public health. International Conference on Weblogs and Social Media [Internet]. $2011 \mathrm{Jul}$ [cited March 15, 2019] 17;20:265-72. Available from https://www. aaai.org/ocs/index.php/ICWSM/ICWSM11/paper/viewFile/2880/3264

6. Vance K, Howe W, Dellavalle RP. Social internet sites as a source of public health information. Dermatol Clin. 2009:27(2):133-6.

7. Thompson L, Rivara FP, Whitehill JM. Prevalence of cannabis-related traffic on twitter, 2012-2013: a content analysis. Cyberpsychol Behav Soc Netw. 2015;18(6):311-9.

8. Cavazos-Rehg PA, Krauss M, Fisher SL, Salyer P, Grucza RA, Bierut LJ. Twitter chatter about marijuana. J Adolesc Health. 2015;56(2):139-45.

9. SocioCultural Research Consultants. Dedoose version 8.0.35, web application for managing, analyzing, and presenting qualitative and mixed method research data. Los Angeles: SocioCultural Research Consultants, LLC; 2018.

10. de Vries H, Elliott MN, Kanouse DE, Teleki SS. Using pooled kappa to summarize interrater agreement across many items. Field Methods. 2008; 20(3):272-82.

11. Cicchetti DV. Guidelines, criteria, and rules of thumb for evaluating normed and standardized assessment instruments in psychology. Psychol Assess. 1994;6(4):284

12. Cabrera-Nguyen EP, Cavazos-Rehg P, Krauss M, Bierut LJ, Moreno MA. Young adults' exposure to alcohol-and marijuana-related content on twitter Journal of studies on alcohol and drugs. 2016:77(2):349-53.

13. Government of Canada. Consumer information Cannabis. Obtained July 27, 2019 from https://www.canada.ca/en/health-canada/services/drugsmedication/cannabis/laws-regulations/regulations-support-cannabis-act/ consumer-information.html. Accessed 29 July.

14. National Conference of State Legislators. State Medical Marijuana Laws. Obtained July 29, 2019 from http://www.ncsl.org/research/health/statemedical-marijuana-laws.aspx\#2. Accessed 29 July.

15. Government of Canada. Cannabis Act Justice Laws Website Obtained July 27, 2019. from https://laws-lois.justice.gc.ca/eng/acts/C-24.5/

16. Burton S, Dadich A, Soboleva A. Competing voices: marketing and countermarketing alcohol on twitter. Journal of Nonprofit \& Public Sector Marketing. 2013;25(2):186-209.

17. Lim MS, Hare JD, Carrotte ER, Dietze PM. An investigation of strategies used in alcohol brand marketing and alcohol-related health promotion on Facebook. Digital health. 2016;2:2055207616647305.

18. Bernoff J, Li C. Harnessing the power of the oh-so-social web. MIT Sloan Manag Rev. 2008;49(3):36.

\section{Publisher's Note}

Springer Nature remains neutral with regard to jurisdictional claims in published maps and institutional affiliations. 